\title{
Two precision medicine predictive tools for six malignant solid tumors: from gene-based research to clinical application
}

\author{
Zhiqiao Zhang ${ }^{\dagger}$ (D) Tingshan $\mathrm{He}^{\dagger}$, Liwen Huang ${ }^{\dagger}$, Yanling Ouyang, Jing Li, Yiyan Huang, Peng Wang \\ and Jianqiang Ding ${ }^{*}$
}

\begin{abstract}
Background: The current study aimed to construct competing endogenous RNA (ceRNA) regulation network and develop two precision medicine predictive tools for colorectal cancer (CRC).

Methods: Differentially expressed (DE) analyses were performed between CRC tissues and normal tissues. A ceRNA regulation network was constructed based on DEIncRNAs, DEmiRNAs, and DEmRNAs.

Results: Fifteen mRNAs (ENDOU, MFN2, FASLG, SHOC2, VEGFA, ZFPM2, HOXC6, KLK10, DDIT4, LPGAT1, BEX4, DENND5B, PHF20L1, HSP90B1, and PSPC1) were identified as prognostic biomarkers for CRC by multivariate Cox regression. Then a Fifteen-mRNA signature was developed to predict overall survival for CRC patients. Concordance indexes were $0.817,0.838$, and 0.825 for 1-, 2- and 3-year overall survival. Patients with high risk scores have worse OS compared with patients with low risk scores.
\end{abstract}

Conclusion: The current study provided deeper understanding of prognosis-related ceRNA regulatory network for CRC. Two precision medicine predictive tools named Smart Cancer Survival Predictive System and Gene Survival Analysis Screen System were constructed for CRC. These two precision medicine predictive tools can provide valuable precious individual mortality risk prediction before surgery and improve the individualized treatment decision-making.

Keywords: Competitive endogenous RNA, Colorectal cancer, mRNA, Overall survival, Prognostic model

\section{Introduction}

Colorectal cancer (CRC) is one of the most prevalent malignant tumors worldwide. There were around 1.1 million newly diagnosed CRC patients and 0.55 million CRC patients died in 2018 [1]. The 5-year overall survival (OS) of CRC patients was less than $12 \%[2,3]$. Due to the poor overall survival, early detection of CRC patients with high mortality risk has important significance for improving the individualized treatment decision-making. Several prognostic models have been developed for CRC patients $[4,5]$. However, the computational formulas of these prognostic models were too complex for clinical

\footnotetext{
*Correspondence: jding18@foxmail.com

'Zhiqiao Zhang, Tingshan He and Liwen Huang were the co-first authors Department of Infectious Diseases, Shunde Hospital, Southern Medical University, No. 1 Jiazi Road, Lunjiao, Shunde District, Foshan 528308, Guangdong Province, China
}

application by patients without calculation tools and medical knowledge. Additional, these prognostic models provided overall survival prediction for different groups, but not individual mortality risk prediction.

From the perspective of precise medicine, a good prognostic model should be able to provide individual mortality risk prediction for specific patient at the individual level. Considering the clinical need of precious individual mortality risk prediction for patients with different tumors, our research team has developed several precision medicine predictive tools for gastric cancer [6] and hepatocellular carcinoma [7]. For individual mortality risk prediction, our precision medicine predictive tools have the following advantages: full-time individual risk prediction, visual illustration, numerical presentation, customizable subgroups, and on-line computing.

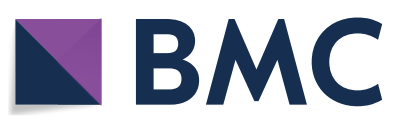

(c) The Author(s) 2019. This article is distributed under the terms of the Creative Commons Attribution 4.0 International License (http://creativecommons.org/licenses/by/4.0/), which permits unrestricted use, distribution, and reproduction in any medium, provided you give appropriate credit to the original author(s) and the source, provide a link to the Creative Commons license, and indicate if changes were made. The Creative Commons Public Domain Dedication waiver (http://creativecommons.org/ publicdomain/zero/1.0/) applies to the data made available in this article, unless otherwise stated. 
So far the molecular biological regulatory mechanism of development and prognosis of tumor remains unclear. Several researches have explored underlying molecular biological regulatory mechanism for different tumors [8-11]. Salmena and collaborators presented an interesting molecular regulatory mechanism named competing endogenous RNAs (ceRNAs) [12]. The lncRNAs can indirectly regulate the expression of mRNAs through binding shared miRNA response elements [13]. Several researches have explored potential molecular regulatory mechanism for CRC [14-16]. Therefore the current research aimed to depict prognosis related ceRNA regulatory network and develop two precision medicine predictive tools for CRC patients.

\section{Materials and methods Study cohort}

RNA sequencing and miRNA sequencing data were obtained from TCGA database. RNA sequencing data contained 14,449 lncRNAs and 20,337 mRNAs whereas miRNA sequencing data contained 1881 miRNAs. Four hundred and twenty-eight CRC patients were included after removing patients without complete survival information. GSE17538 dataset were downloaded from GEO database. GSE17538 dataset involved 231 CRC patients and 23,328 RNAs (GPL570 platform). The original read count values in TCGA dataset were normalized by $\log 2$ transformation. The gene background file (Gencode.v29 supplied by The European Bioinformatics Institute of The European Molecular Biology Laboratory (EMBL-EBI) database) was used for gene symbol annotation.
Differentially expressed analyses and regulatory network The original RNA data were processed by "edgeR" package, with a defined $P$ of 0.05 and a ratio of 1.5 times between tumor and non-tumor tissues [17]. The original miRNA sequencing data were processed by "limma" package [18]. First, the interaction associations between lncRNAs and miRNAs were identified in miRcode database [19]. Second, miRTarBase [20], miRDB [21], and TargetScan [22] were searched for miRNA-targeted mRNAs. The ceRNA network was visualized by Cytoscape v3.6.1 [23].

\section{Statistical analyses and artificial intelligence algorithms}

Random survival forest, Multi-task logistic regression, and Cox survival regression algorithms were carried out according to the algorithms suggested in the original articles [24-29]. Statistical analyses were carried out through SPSS Statistics 19.0 (SPSS Inc.,USA). Other analyses were carried out by $\mathrm{R}$ version 3.5.2 with corresponding packages. $P$ value $<0.05$ was defined as statistically significant.

\section{Results}

\section{Baseline characteristics}

TCGA dataset contained 428 CRC patients and GSE17538 dataset contained 231 CRC patients. The clinical information of included patients was shown in Table 1. The mortality of GSE17538 dataset was $40.3 \%$ (93/231), which was significantly higher than $22.9 \%$ $(98 / 428)$ of TCGA dataset. There were significant differences in terms of survival time and pathological stage,

Table 1 Clinical features of colorectal cancer patients

\begin{tabular}{|c|c|c|c|}
\hline & TCGA cohort & GSE17538 & $P$-value \\
\hline Number $[\mathrm{n}]$ & 428 & 231 & NA \\
\hline Death $[n(\%)]$ & $98(22.9)$ & $93(40.3)$ & $<0.001$ \\
\hline Total survival time (mean \pm SD, month) & $29.8 \pm 25.6$ & $47.6 \pm 30.6$ & $<0.001$ \\
\hline Survival time for dead patients (month) & $23.3 \pm 22.7$ & $26.8 \pm 22.0$ & $<0.001$ \\
\hline Survival time for living patients (month) & $31.7 \pm 26.1$ & $61.7 \pm 27.5$ & $<0.001$ \\
\hline Age (mean $\pm S D$, year) & $66.5 \pm 13.0$ & $64.8 \pm 13.4$ & 0.100 \\
\hline Male $[(n) \%]$ & $230(53.7)$ & $121(52.4)$ & 0.739 \\
\hline AJCC stage (IV/III/II/I/NA) & $60 / 124 / 163 / 70 / 11$ & $56 / 75 / 72 / 28 / 0$ & $<0.001$ \\
\hline AJCC PT (T1/TO/NA) & $51 / 294 / 72 / 11 / 0$ & NA & NA \\
\hline AJCC PN (N2/N1/N0/NA) & $76 / 103 / 249 / 0$ & NA & NA \\
\hline AJCC PM (M2/M1/M0/NA) & $47 / 60 / 315 / 6$ & NA & NA \\
\hline Lymphovascular invasion (yes/no/NA) & $148 / 237 / 43$ & NA & NA \\
\hline Vascular invasion (yes/no/NA) & $89 / 281 / 58$ & NA & NA \\
\hline Residual tumor (3/2/1/0/NA) & $23 / 21 / 4 / 307 / 73$ & NA & NA \\
\hline Perineural invasion (yes/no/NA) & $45 / 126 / 257$ & NA & NA \\
\hline Grade (1/0/NA) & NA & $55 / 144 / 32$ & NA \\
\hline
\end{tabular}

SD standard deviation, NA missing data, AJCC American Joint Committee on Cancer 
whereas there were no significant differences in terms of age and gender.

\section{Differentially expressed analyses}

Differential expression analysis could identify genes with significant differences in expression levels between normal samples and tumor samples. Compared with normal tissues, 3005 lncRNAs (2224 up-regulated and 781 downregulated), 332 miRNAs (246 up-regulated and 86 downregulated), and 6713 mRNAs (4087 up-regulated and 2626 down-regulated) were identified in CRC tissues. The volcano plots for differentially expressed RNAs were shown in Additional file 1: Figure S1.

\section{Screening of prognostic mRNAs}

Univariate Cox regression was used to explore potential prognostic biomarkers for CRC. Out of previous differentially expressed mRNAs, there were 2504 mRNAs identified as prognostic biomarkers for CRC. Out of 2504 potential prognostic biomarkers, there were 1371 risk factors and 1133 protective factors.

\section{Development of ceRNA network}

The miRNA- targeted mRNAs that could be searched in three above databases were defined as the
miRNA-targeted mRNAs. Third, these miRNA-targeted mRNAs were intersected with previous prognostic mRNAs for development of ceRNA network. Finally, the ceRNA network, consisting of 14 lncRNAs, 29 miRNAs, and 79 mRNAs, were constructed for CRC. The ceRNA network was visualized in Fig. 1 by Cytoscape v3.6.1. This ceRNA network depicted potential regulatory relations among lncRNAs, miRNAs, and mRNAs, and was helpful to understand the potential mechanisms of tumor prognosis.

\section{Functional enrichment analyses}

Functional enrichment analyses were performed based on previous prognostic mRNAs in ceRNA network and identified 26 enriched Gene Ontology (GO) terms. The top 15 enriched GO terms were shown in Fig. 2a. The prognostic mRNAs were mainly enriched in transcription factor activity, RNA polymerase II proximal promoter sequence-specific DNA binding, regulation of MAP kinase activity, regulation of protein serine/threonine kinase activity, inactivation of MAPK activity, MAP kinase tyrosine/serine, MAP kinase phosphatase activity, protein tyrosine/serine, E-box binding, protein tyrosine phosphatase activity, and negative regulation of MAP kinase activity. The top KEGG pathways (Fig. 2b) were

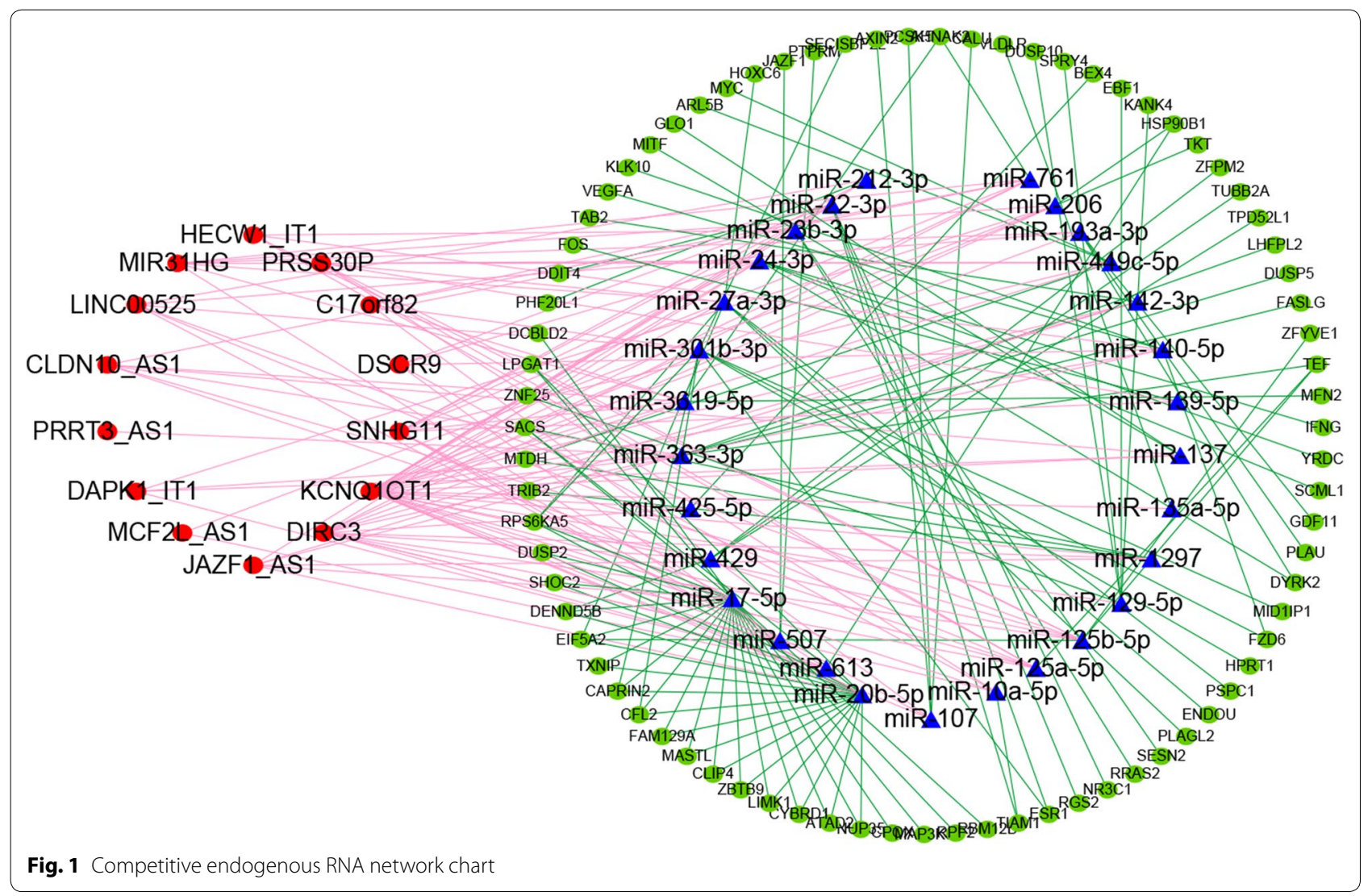



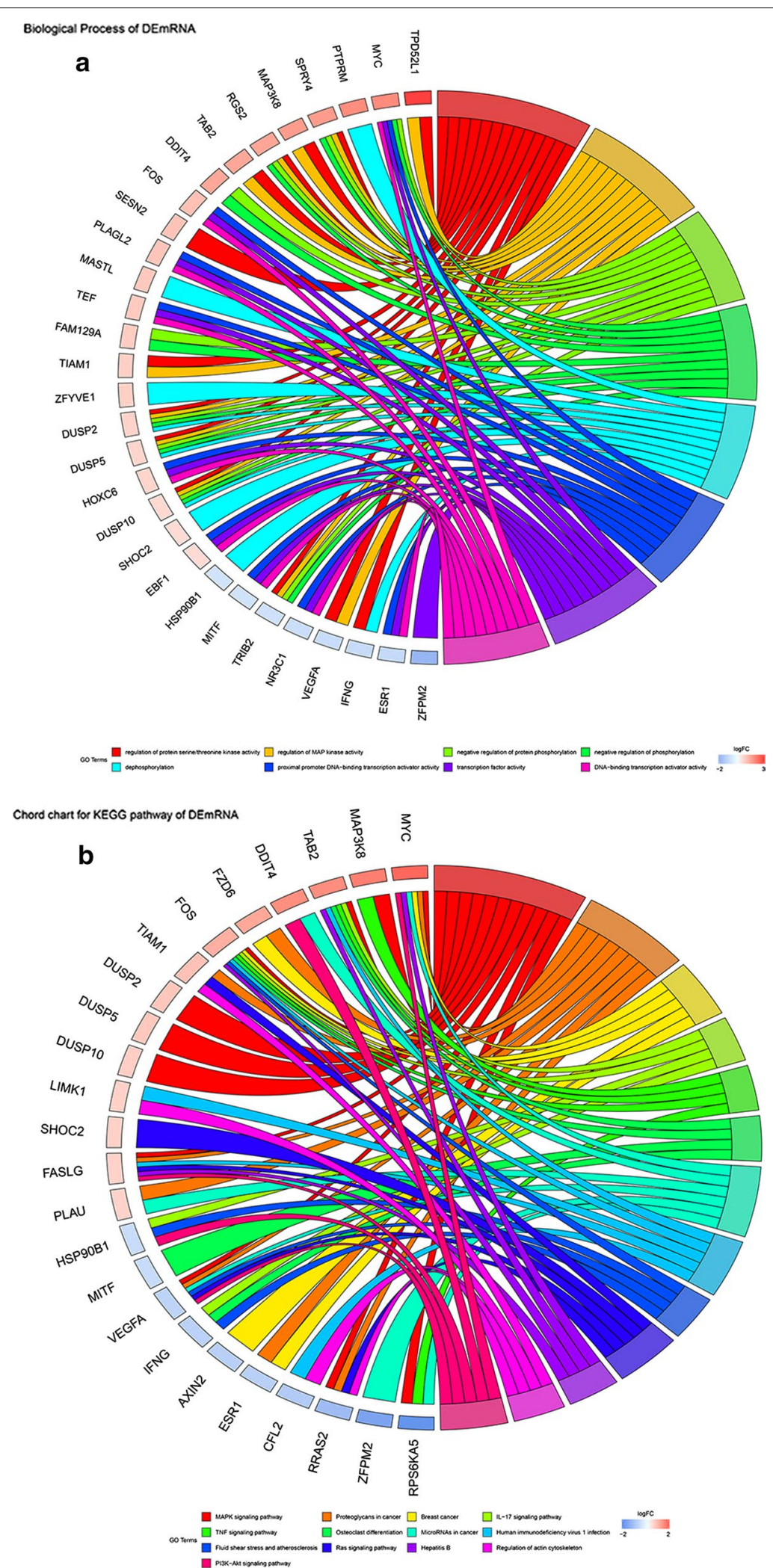

Fig. 2 Chord chart of prognostic mRNAs: a GO terms; b KEGG pathways 
mainly enriched in MAPK signaling pathways, Proteoglycans in cancer, Breast cancer, IL-17 signaling pathway, TNF signaling pathway, Osteaclast differentiation, MicorRNAs in cancer, Human immunodeficiency virus 1 infection, Fluid shear stress and atherosclesosis, Ras signaling pathway, Hepatitis B, Regulation of action cytoskeleton, PI3K-Akt signaling pathway. These biological functions were helpful to understand the roles of these genes in tumor prognosis. The further circular visualization chart was presented in Fig. 3.

\section{Construction of predictive model}

Multivariate Cox regression analyses were used to screen independent risk factors for tumor prognosis. Fifteen mRNAs were used to develop a predictive model for CRC (Table 2). The formula for predictive model was as follows: The risk score $=(-4.273 *$ ENDOU $)+(-1.48$ $9 *$ MFN2 $)+(-1.243 *$ FASLG $)+(-0.904 *$ SHOC2 $)+$ $(-0.834$ * VEGFA $)+(-0.690 *$ ZFPM 2$)+(0.249 *$ HOX C6 $)+(0.446 *$ KLK10 $)+(0.672 *$ DDIT4 $)+(0.705 *$ LPG $\mathrm{AT} 1)+(0.711 * \mathrm{BEX} 4)+(1.038 * \mathrm{DENND} 5 \mathrm{~B})+(1.065$ * PHF20L1 $)+(1.093 *$ HSP90B1 $)+(1.146$ * PSPC1). The prognostic nomogram chart was shown in Fig. 4.

\section{Survival curve analyses}

Survival curve analyses were performed to explore the survival influences of included mRNAs (Additional file 1: Figure S2). Kaplan-Meier curves indicated that patients with high expression had significantly poor overall survival than that with low expression for these 15 mRNAs $(P<0.05)$. Comparison of Kaplan-Meier curves supported that these 15 genes were associated with overall survival in CRC patients.

\section{Predictive performance in model dataset}

CRC patients were divided into high risk subgroup and low risk subgroup according to median risk score. Figure $5 \mathrm{a}$ demonstrated that there was significant difference between two subgroups for OS $(P<0.001)$. Concordance indexes were $0.817,0.838$, and 0.825 respectively for 1 -year, 2 -year, and 3-year OS (Fig. 5b). Calibration curves for OS were presented in Fig. 6, indicating a good agreement between predicted mortality and actual mortality for 1-year, 2-year, and 3-year OS.

\section{Predictive performance in validation dataset}

Kaplan-Meier plot (Fig. 7a) demonstrated that OS in high risk subgroup was significantly worse than that in low risk subgroup $(P<0.05)$. Concordance indexes were $0.773,0.824$, and 0.801 for 1-year, 2-year, and 3-year OS (Fig. 7b). Calibration curves for OS were depicted in Fig. 8, demonstrating that the predicted mortality was in good agreement with the actual mortality.

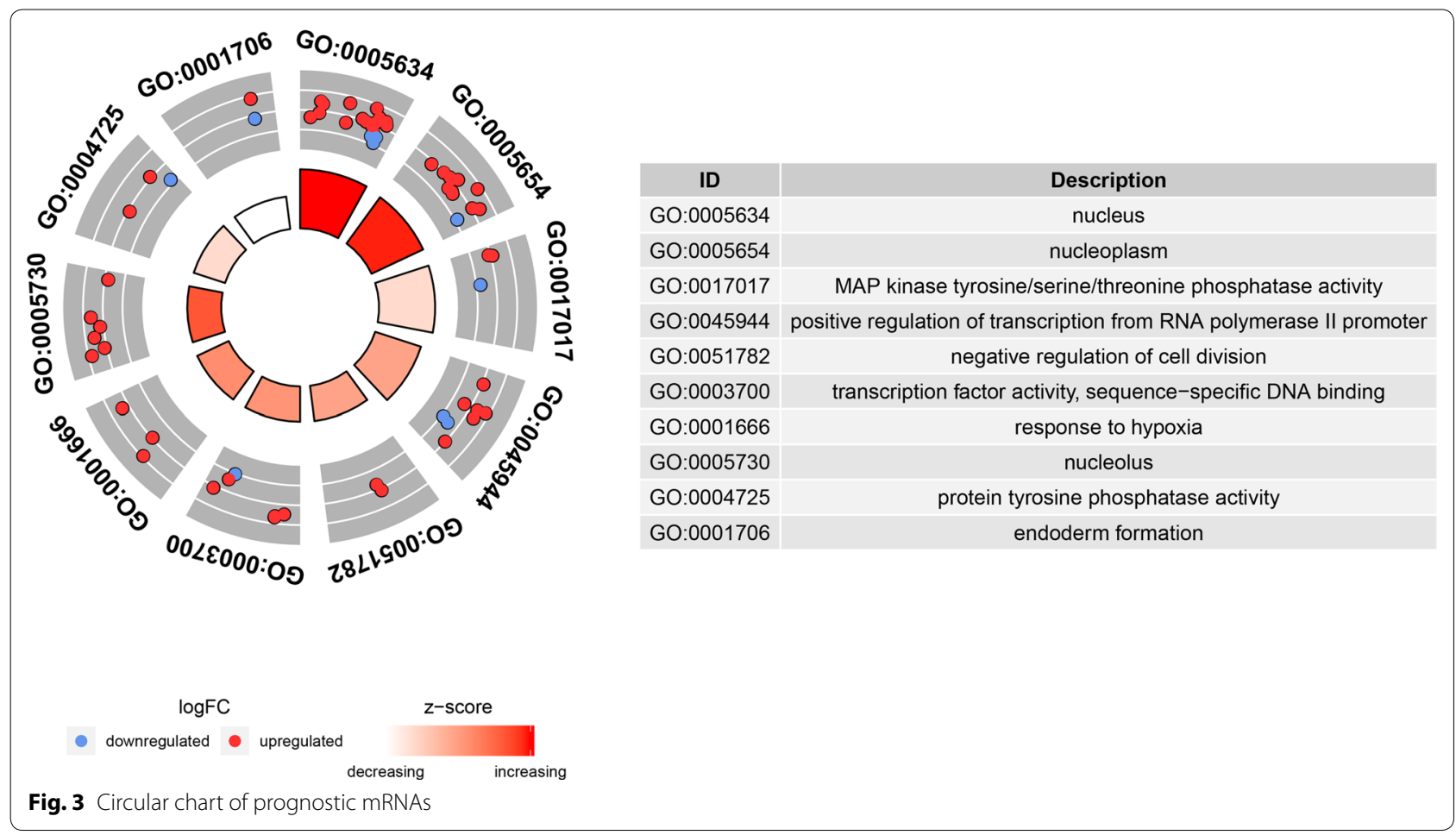


Table 2 Model information of prognostic mRNAs

\begin{tabular}{|c|c|c|c|c|c|c|c|}
\hline \multirow[t]{2}{*}{ Gene } & \multicolumn{3}{|c|}{ Univariate analysis } & \multicolumn{4}{|c|}{ Multivariate analysis } \\
\hline & HR & $95 \% \mathrm{Cl}$ & $P$-value & Coefficient & HR & $95 \% \mathrm{Cl}$ & $P$-value \\
\hline ENDOU & 0.642 & $0.425-0.972$ & 0.036 & -4.273 & 0.014 & $0.004-0.047$ & $<0.001$ \\
\hline MFN2 & 0.632 & $0.417-0.957$ & 0.030 & -1.489 & 0.226 & $0.125-0.408$ & $<0.001$ \\
\hline FASLG & 0.560 & $0.368-0.853$ & 0.007 & -1.243 & 0.288 & $0.098-0.846$ & 0.024 \\
\hline SHOC2 & 1.544 & $1.023-2.333$ & 0.039 & -0.904 & 0.405 & $0.179-0.917$ & 0.030 \\
\hline VEGFA & 1.569 & $1.037-2.373$ & 0.033 & -0.834 & 0.434 & $0.226-0.833$ & 0.012 \\
\hline ZFPM2 & 1.805 & $1.183-2.753$ & 0.006 & -0.690 & 0.501 & $0.363-0.693$ & $<0.001$ \\
\hline HOXC6 & 1.567 & $1.037-2.368$ & 0.033 & 0.249 & 1.283 & $1.064-1.547$ & 0.009 \\
\hline KLK10 & 1.593 & $1.053-2.409$ & 0.028 & 0.446 & 1.562 & $1.161-2.100$ & 0.003 \\
\hline DDIT4 & 1.710 & $1.126-2.596$ & 0.012 & 0.672 & 1.959 & $1.319-2.908$ & 0.001 \\
\hline LPGAT1 & 1.570 & $1.039-2.371$ & 0.032 & 0.705 & 2.023 & $1.199-3.415$ & 0.008 \\
\hline BEX4 & 1.795 & $1.185-2.721$ & 0.006 & 0.711 & 2.036 & $1.521-2.725$ & $<0.001$ \\
\hline DENND5B & 1.527 & $1.010-2.308$ & 0.045 & 1.038 & 2.824 & $1.155-6.909$ & 0.023 \\
\hline PHF20L1 & 1.562 & $1.028-2.373$ & 0.037 & 1.065 & 2.901 & $1.162-7.241$ & 0.022 \\
\hline HSP90B1 & 1.959 & $1.291-2.972$ & 0.002 & 1.093 & 2.982 & $1.561-5.696$ & 0.001 \\
\hline PSPC1 & 1.863 & $1.228-2.825$ & 0.003 & 1.146 & 3.144 & $1.682-5.877$ & $<0.001$ \\
\hline
\end{tabular}

$H R$ hazard ratio, $\mathrm{Cl}$ confidence interval

\section{Independence assessment of predictive model}

In model cohort, prognostic signature and pathological stage were independent influence factors for OS (Table 3). In validation cohort, prognostic signature, pathological stage, and age were identified as independent influence factors for OS. Decision curves and clinical impact curve for OS were presented in Additional file 1: Figure S3, suggesting that the clinical efficacy of the current prognostic model was superior to pathological stage and age.

\section{Smart Cancer Survival Predictive System}

We developed a precision medicine predictive tool named Smart Cancer Survival Predictive System, providing a novel convenient on-line calculator for prediction of OS. Smart Cancer Survival Predictive System (Fig. 9) is available at: https://zhangzhiqiao6.shinyapps.io/Smart_ cancer_predictive_system_11_CRC_B1003/. Smart Cancer Survival Predictive System provided three individual mortality risk curves predicted by Random survival forest algorithm, Multitask logistic regression algorithm, and Cox survival regression algorithm according to the calculation formula in ogininal articles.

\section{Gene Survival Analysis Screen System}

To further explore survival curves of previous prognostic genes in different gender and pathological stage subgroups, we developed a new online program named Gene Survival Analysis Screen System (Fig. 10). Gene Survival Analysis Screen System is available at: https://zhang
zhiqiao5.shinyapps.io/Gene_Survival_Subgroup_Analy sis_B1003/. Gene Survival Analysis Screen System provided seven tumor datasets for exploration research and allowed users to select tumor type, gender, and stage, which were the important influence factors to the prognosis.

\section{Clinical application in other tumors}

The current study download five different tumor datasets from TCGA database as external validation datasets to explore the clinical application of the current prognostic model. Figure 11 displayed the diagnostic accuracy of the current prognostic model in five tumors, including hepatocellular carcinoma $(n=348)$, breast cancer $(n=1030)$, gastric cancer $(n=265)$, lung cancer $(n=494)$, and ovarian cancer $(n=370)$.

\section{Predictive performance in five malignant solid tumors}

To further explore the predictive performance of the current prognostic model, we externally validated the current prognostic model in a super merge dataset $(n=2507)$, which including five malignant solid tumors (hepatocellular carcinoma, breast cancer, gastric cancer, lung cancer, and ovarian cancer). Kaplan-Meier plot (Fig. 12a) demonstrated that OS in high risk subgroup was significantly worse than that in low risk subgroup $(P<0.05)$. Concordance indexes were $0.663,0.639$, and 0.655 for 1-year, 2-year, and 3-year OS (Fig. 12b). Calibration curves for OS were depicted in Fig. 13, demonstrating that the predicted mortality was in good agreement with the actual mortality. 


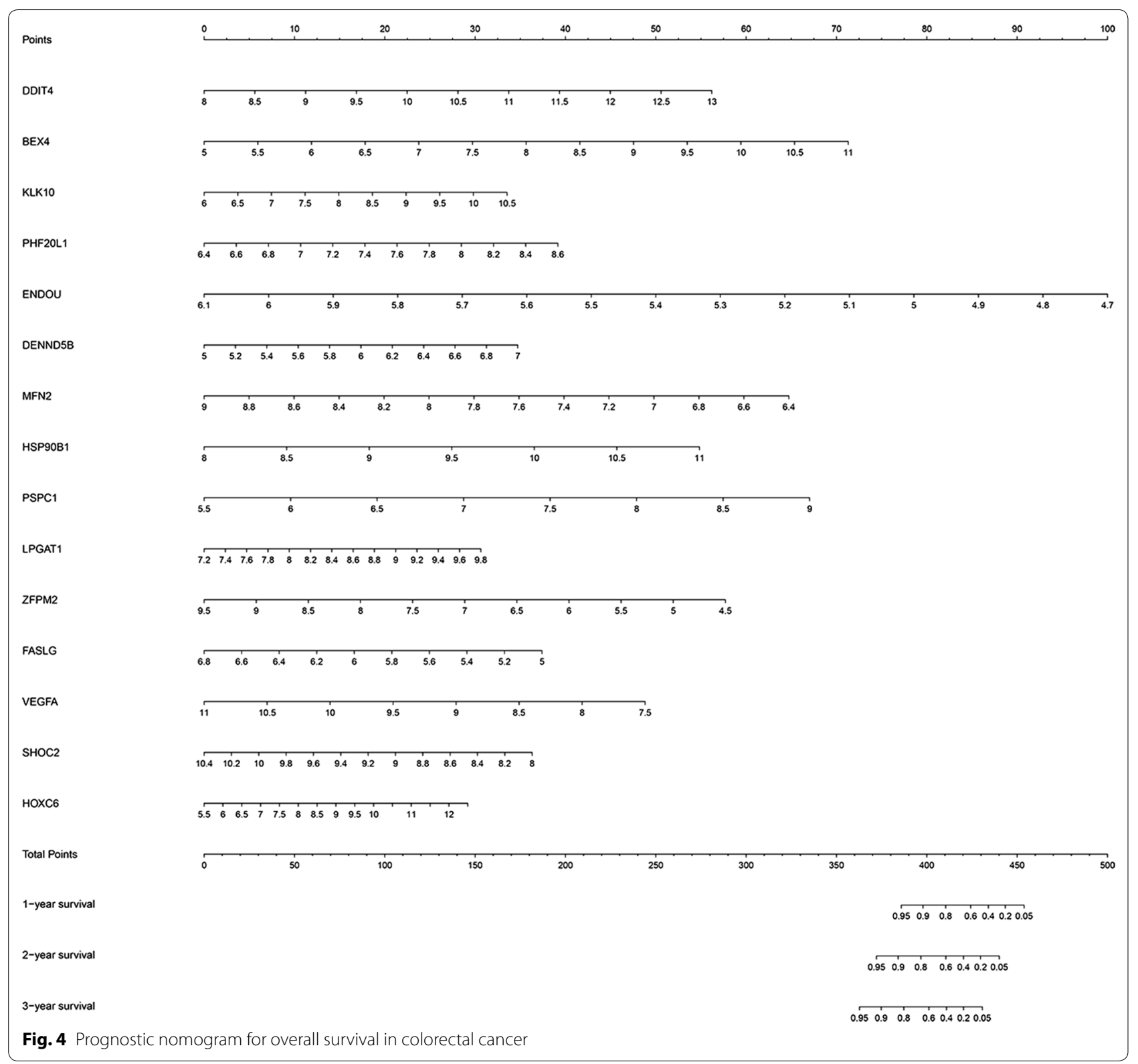

\section{Discussion}

The current research constructed a prognosis-related ceRNA network for CRC. A prognostic nomogram was developed for OS based on prognostic mRNAs in ceRNA network. The current prognostic model could discriminate high risk patients from low risk patients for different time points. Meanwhile, two novel precision medicine predictive tools were developed to provide convenient on-line calculation for prediction of OS in CRC patients. Smart Cancer Survival Predictive System provided three individual mortality risk curves predicted by Random survival forest algorithm, Multitask logistic regression algorithm, and Cox survival regression. Gene
Survival Analysis Screen System provided survival curve comparison and multivariable survival analysis results. Smart Cancer Survival Predictive System and Gene Survival Analysis Screen System could provide precious individualized mortality risk prediction for CRC patients.

The current study screened differentially expressed RNAs between CRC tissues and normal tissues and then constructed a prognosis-related ceRNA regulatory network for CRC. Based on mRNAs in ceRNA regulatory network, the current study further identified independent prognostic biomarkers for overall survival in CRC patients. Through ceRNA regulatory network, the 

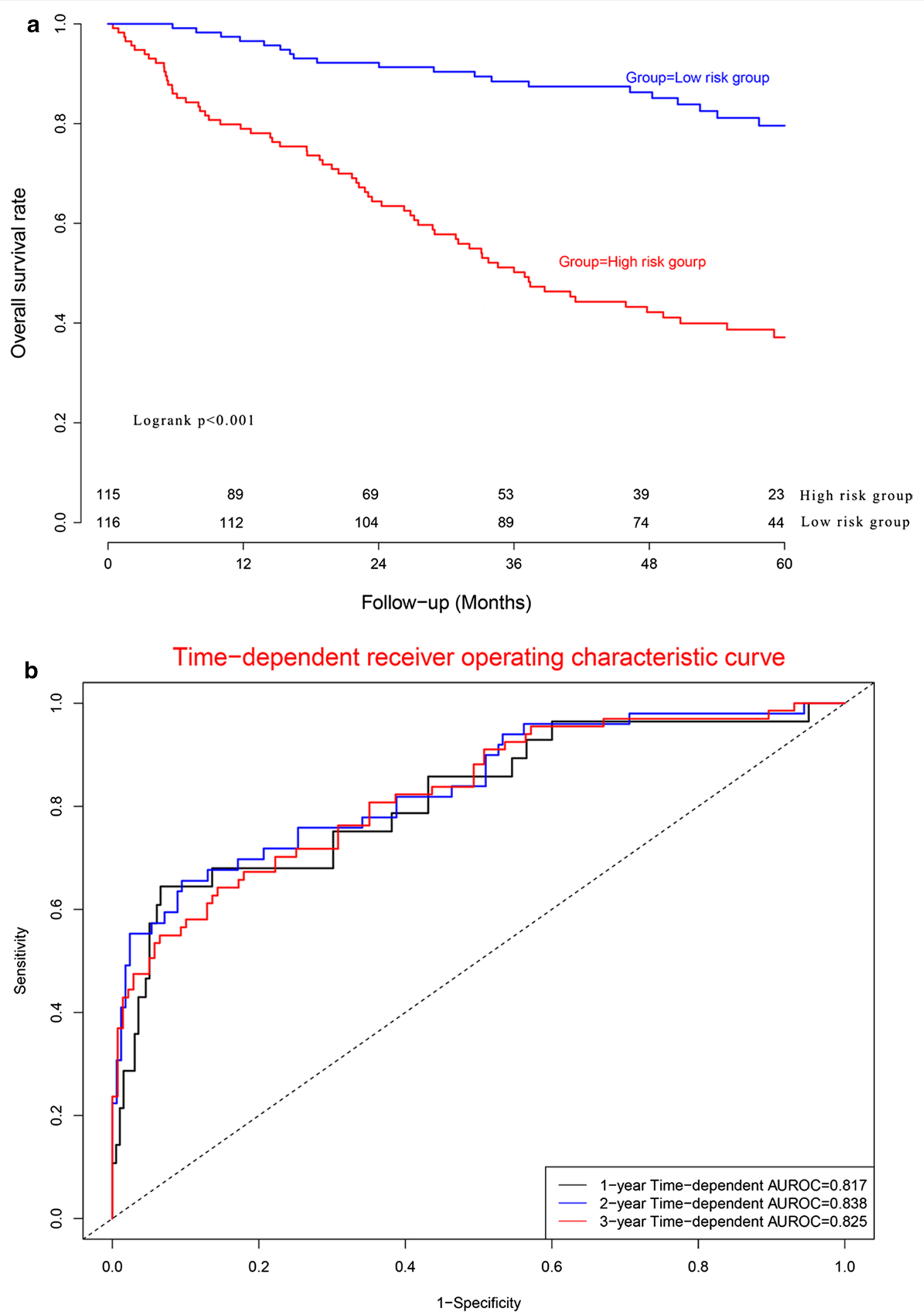

Fig. 5 Clinical performance in model cohort: a survival curve plot; $\mathbf{b}$ time-dependent receiver operating characteristic curve plot

regulatory relationship among lncRNA-miRNA-mRNA was depicted, providing post-transcriptional biological regulatory pathway information for CRC. GO term and
KEGG pathway analyses were helpful to further understand the biological function and molecular regulatory pathway of prognostic mRNAs in ceRNA network. The 

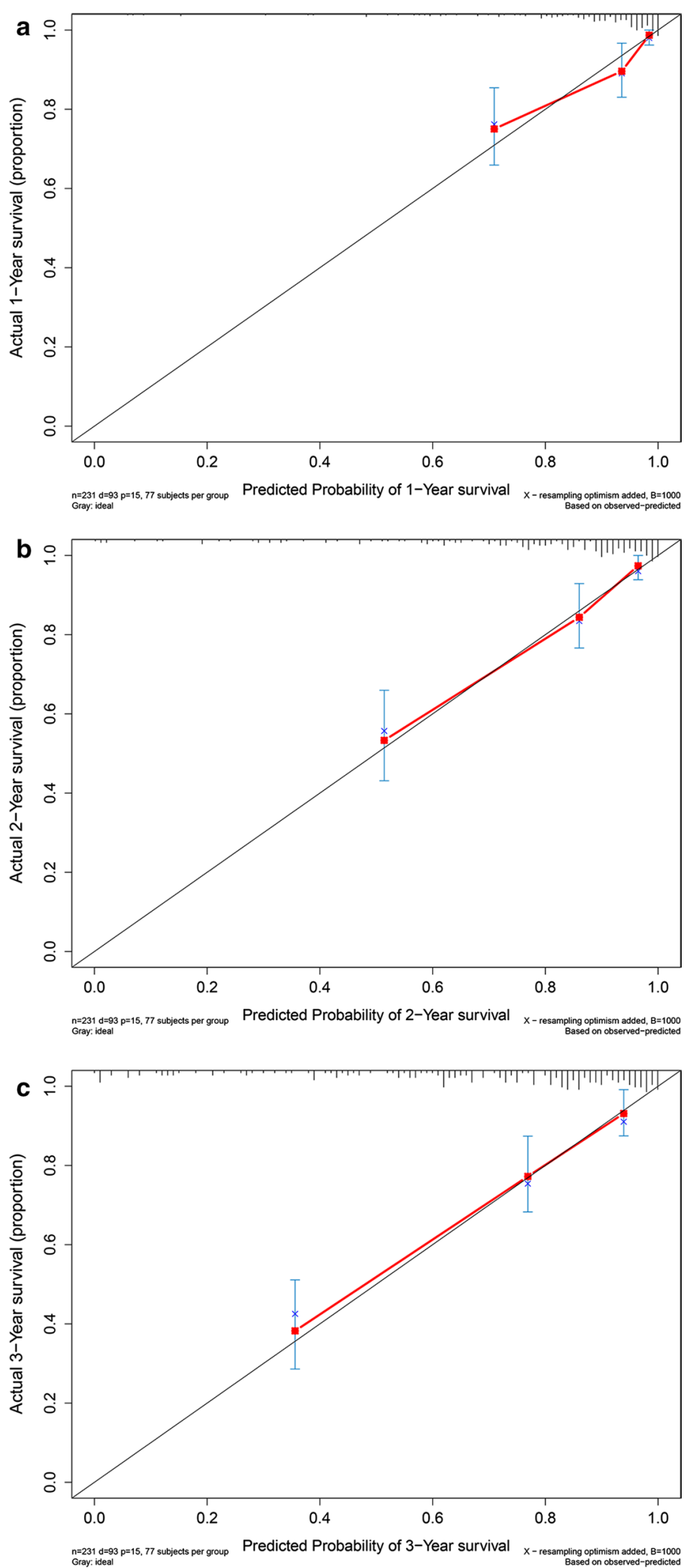

Fig. 6 Calibration curves for model cohort. a Calibration curve for 1-year survival; b calibration curve for 2-year survival; c calibration curve for 3-year survival 

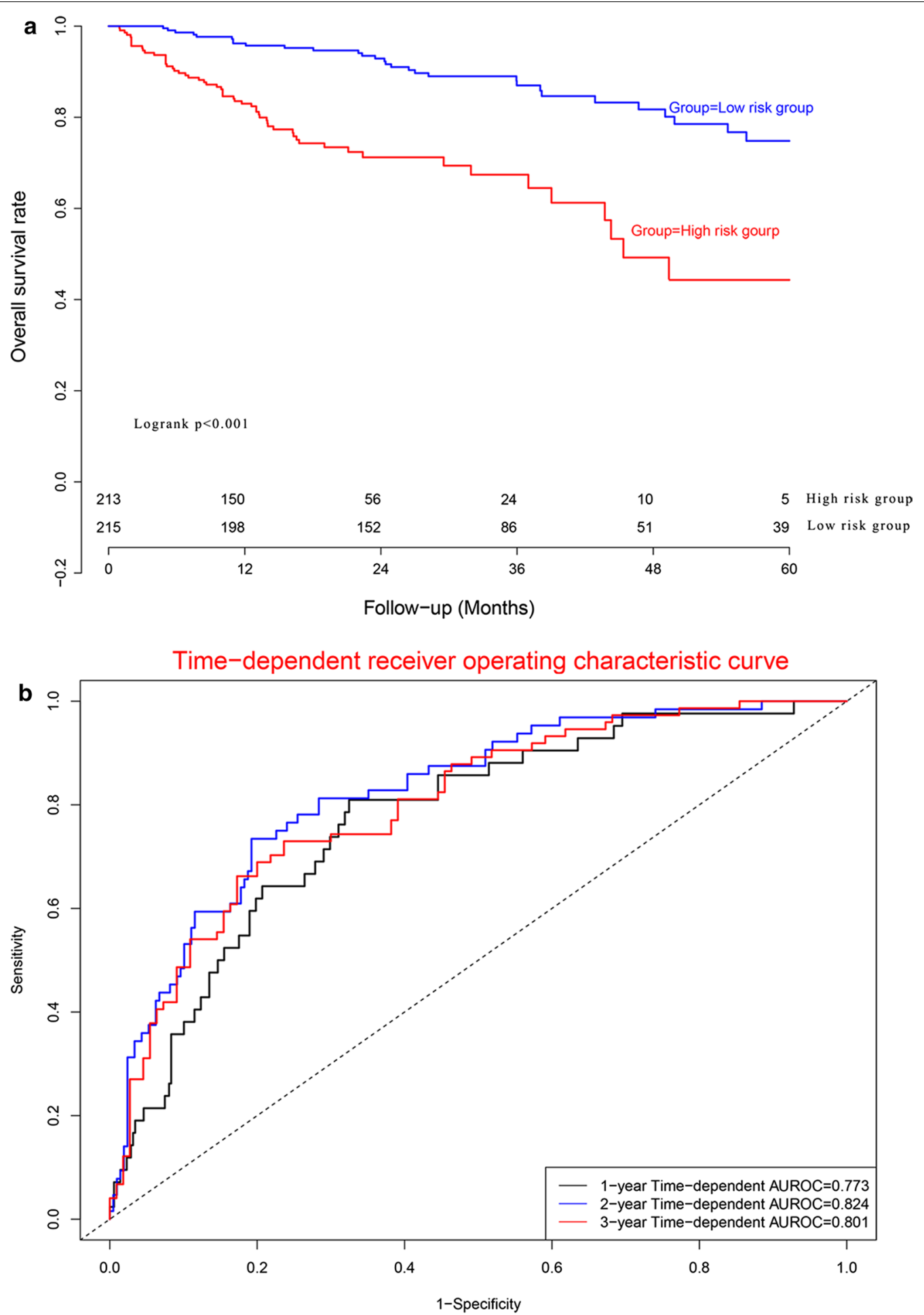

Fig. 7 Clinical performance in validation cohort: a survival curve plot; $\mathbf{b}$ time-dependent receiver operating characteristic curve plot

fifteen prognostic mRNAs were identified as independent prognostic biomarkers for overall survival in CRC patients by multivariable Cox regression. The ceRNA regulatory network and functional enrichment analyses provided potential biological prognostic indicators and therapeutic targets for future researches.

Mitofusin 2 (MFN2) promotes cell proliferation and invasiveness in gastric cancer [30]. Lin et al. reported 

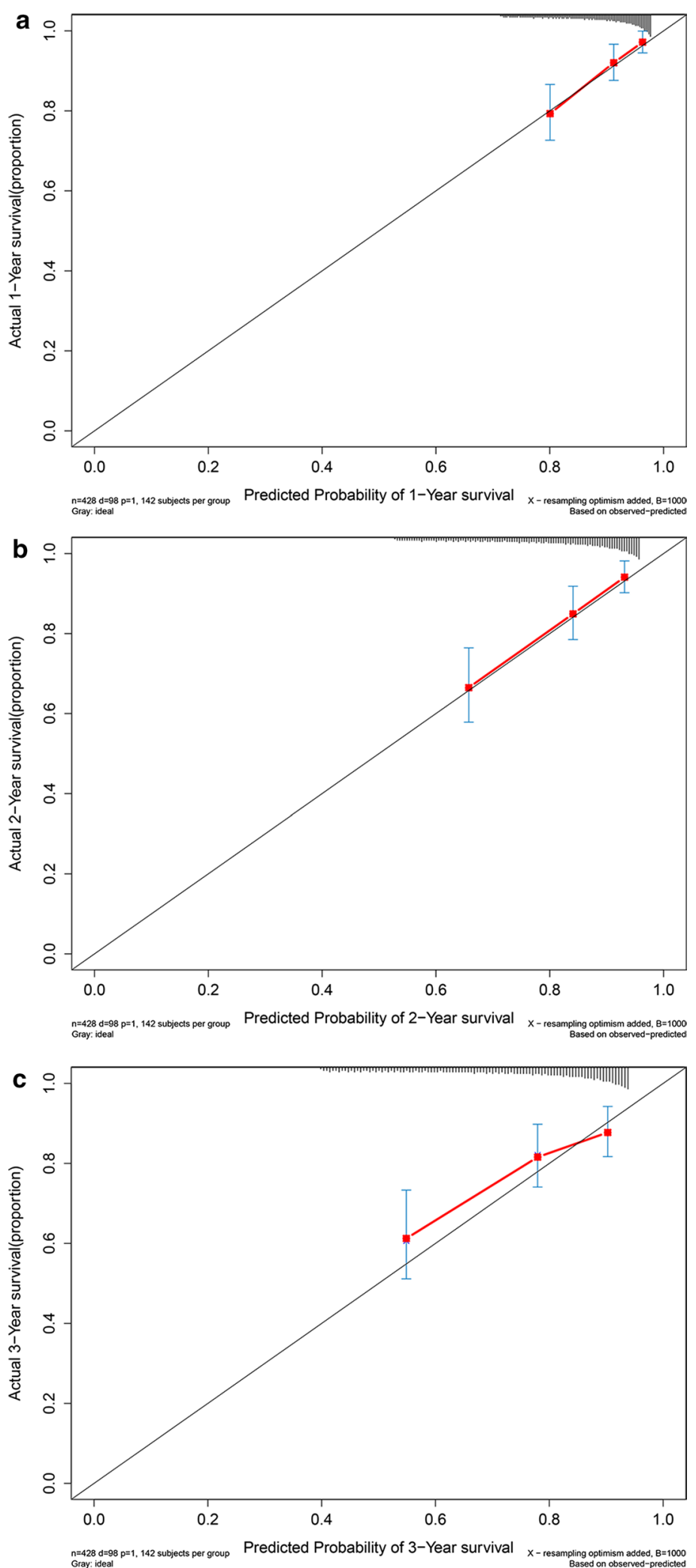

Fig. 8 Calibration curves for validation cohort. a Calibration curve for 1-year survival; b calibration curve for 2-year survival; c calibration curve for 3-year survival 
Table 3 Independence assessment of prognostic model

\begin{tabular}{|c|c|c|c|c|c|c|c|}
\hline & \multicolumn{3}{|c|}{ Univariate analysis } & \multicolumn{4}{|c|}{ Multivariate analysis } \\
\hline & HR & $95 \% \mathrm{Cl}$ & $P$-value & coefficient & HR & $95 \% \mathrm{Cl}$ & $P$-value \\
\hline \multicolumn{8}{|l|}{ Model group } \\
\hline Gender (male/female) & 0.883 & $0.586-1.33$ & 0.551 & -0.247 & 0.781 & $0.508-1.202$ & 0.261 \\
\hline Age (high/low) & 0.591 & $0.381-0.917$ & 0.018 & 0.161 & 1.175 & $0.765-1.804$ & 0.463 \\
\hline AJCC stage (IV, III/II, I) & 0.323 & $0.21-0.498$ & $<0.001$ & 1.196 & 3.306 & $1.979-5.522$ & $<0.001$ \\
\hline Prognostic model (high/low) & 6.48 & $2.037-20.61$ & $<0.001$ & 1.499 & 4.476 & $2.706-7.403$ & $<0.001$ \\
\hline \multicolumn{8}{|l|}{ Validation group } \\
\hline Gender (male/female) & 0.993 & $0.66-1.496$ & 0.975 & -0.089 & 0.915 & $0.608-1.379$ & 0.672 \\
\hline Age (high/low) & 0.942 & $0.627-1.415$ & 0.773 & 0.712 & 2.037 & $1.330-3.120$ & $<0.001$ \\
\hline AJCC stage (IV, III/II, I) & 0.270 & $0.163-0.448$ & $<0.001$ & 1.206 & 3.341 & $2.185-5.107$ & $<0.001$ \\
\hline Prognostic model (high/low) & 0.210 & $0.129-0.342$ & $<0.001$ & -1.228 & 0.293 & $0.189-0.453$ & $<0.001$ \\
\hline
\end{tabular}

AJCC The American Joint Committee on Cancer, HR hazard ratio, Cl confidence interval

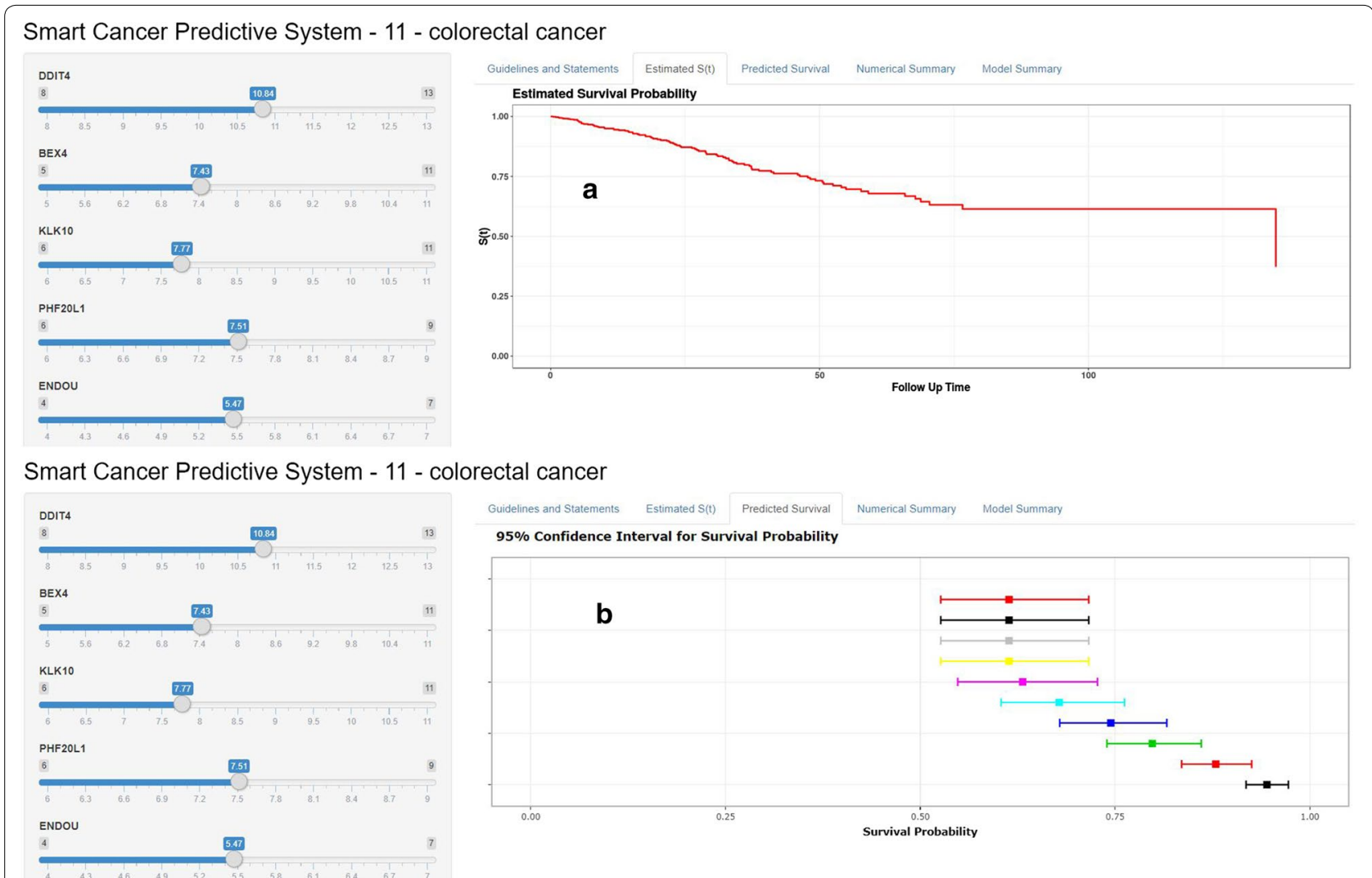

Fig. 9 Home page of Smart Cancer Survival Predictive System. a Survival curves display page; b quantitative display page

that miRNA-30d can regulate cancer angiogenesis and cancer proliferation through MYPT1/c-JUN/VEGFA pathway [31]. Liu et al. reported that ZFPM2 Antisense RNA 1 (ZFPM2-AS1) promotes tumor proliferation and inhibits apoptosis through regulating miR-137 in renal cell cancer [32]. Li et al. reported that Homeobox
C6 (HOXC6) promotes invasion via EMT pathway in hepatocellular carcinoma [33]. Petraki et al. reported that Kallikrein Related Peptidase 10 (KLK10) expression is associated with overall survival in CRC [34]. Xu et al. reported that MiR-199b-5p can promote tumor proliferation through regulating KLK10 in cervical 
Gene_Survival_Subgroup_Analysis_B1003

Step 1 : Select dataset

Using testing dataset (.x|sx) or upload a new dataset $(. x \mid s x)$ ?

Testing dataset (.xlsx)

Step 2 : Select subgroup Subgroup

10

Patient gender

10

Tumor stage

100233

$\downarrow$ Download testing dataset (.csv)

Kaplan-Meier survival curve

Survival status variable

os

Survival time variable

OS_MONTH

Define time unit \begin{tabular}{l|l|l} 
Original data display & Survival curve analysis Univariate Cox survival analysis table Guidelines and Statements
\end{tabular} a
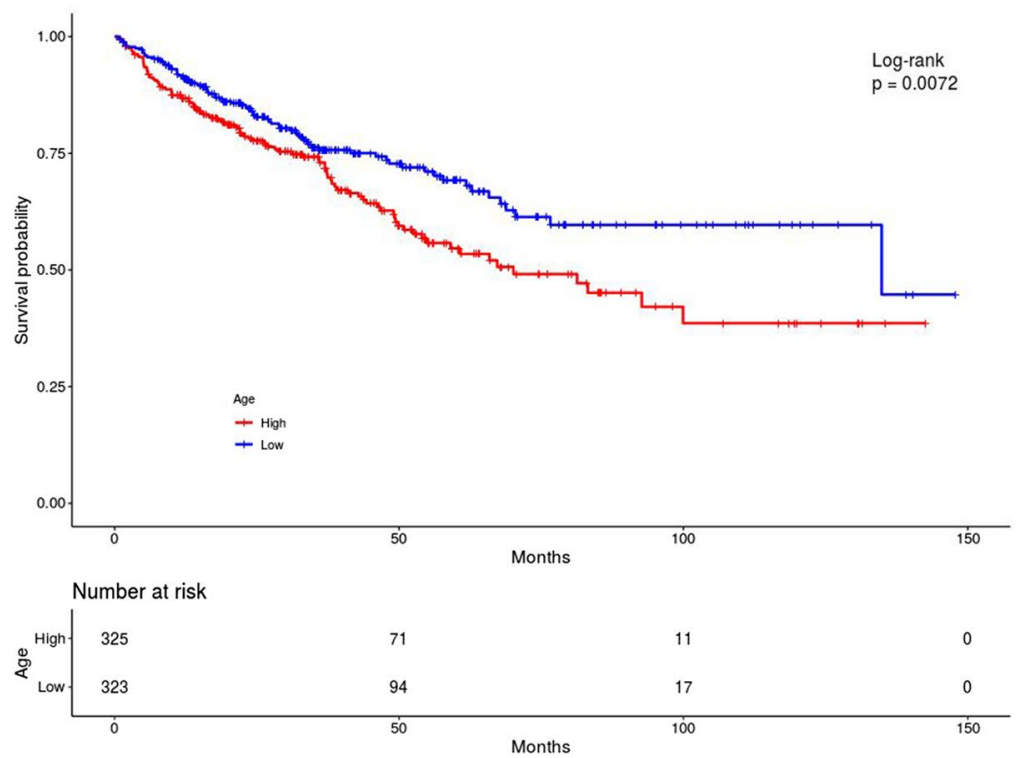

Gene_Survival_Subgroup_Analysis_B1003

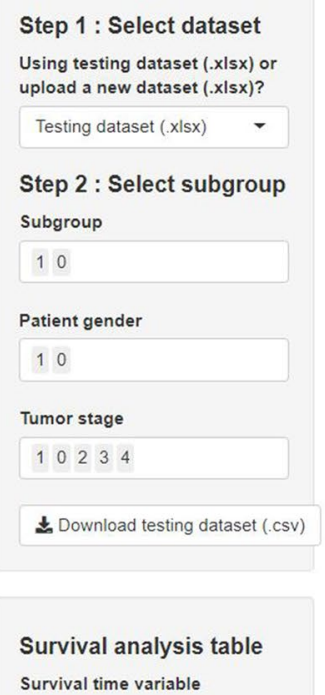

Original data display Survival curve analysis Univariate Cox survival analysis table Guidelines and Statements

\section{Univariate survival analysis table b}

Show 10 entries

\begin{tabular}{llll} 
Variable & Category & Number & Hazard Ratio (95\% confidence interval) \\
\hline Gender & 1 & 344 & $1.067(0.8-1.424)$ \\
\hline & 0 & 304 & \\
\hline Age & Low & 323 & $0.673(0.503-0.9)$ \\
\hline Stage & High & 325 & \\
\hline & 2 & 235 & $2.086(0.981-4.437)$ \\
\hline & 3 & 199 & $3.551(1.696-7.431)$ \\
\hline & 4 & 116 & $12.426(5.99-25.779)$ \\
\hline
\end{tabular}

Showing 1 to 8 of 8 entries

Survival time variable

Fig. 10 Home page of Gene Survival Analysis Screen System. a Survival curves display page; b Cox survival analysis display page

cancer [35]. Liu et al. reported that Heat Shock Protein 90 Beta Family Member 1 (HSP90B1) is significantly related with worse overall survival in lung cancer [36]. Cawthorn et al. reported that up-regulated HSP90B1 is related with distant metastasis [37]. Kessler et al. reported that Paraspeckle Component 1 (PSPC1) is significantly related with poor prognosis in hepatocellular carcinoma [38]. The results in current study further supported the credibility of these findings above in previous researches. 

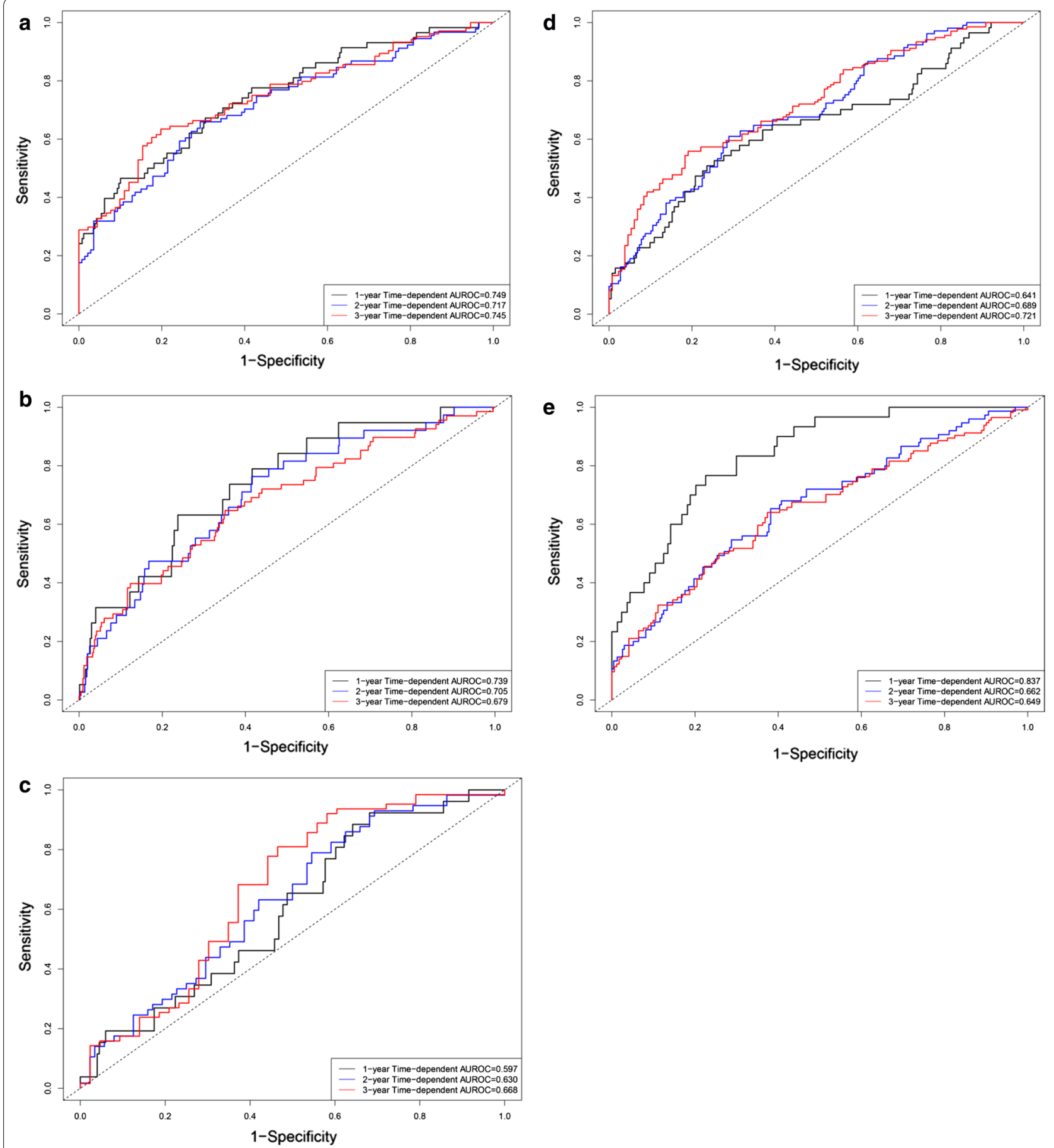

Fig. 11 Time-dependent receiver operating characteristic curve plots. a Hepatocellular carcinoma; b breast cancer; c gastric cancer; $\mathbf{d}$ lung cancer; e ovarian cancer

High quality samples and standardized RNA extraction technology are helpful to improve the quality and reliability of research data. According to the Standard Operating Procedure suggested by TCGA database, for gene sequencing sample, conventional methods of fixation and heating of biopsies may lead to inactivation of antigens and genetic material in tissues, therefore frozen sections was recommended in TCGA database. Samples should be snap-frozen and stored in cryovials in liquid nitrogen vapor and should not allow tissues 

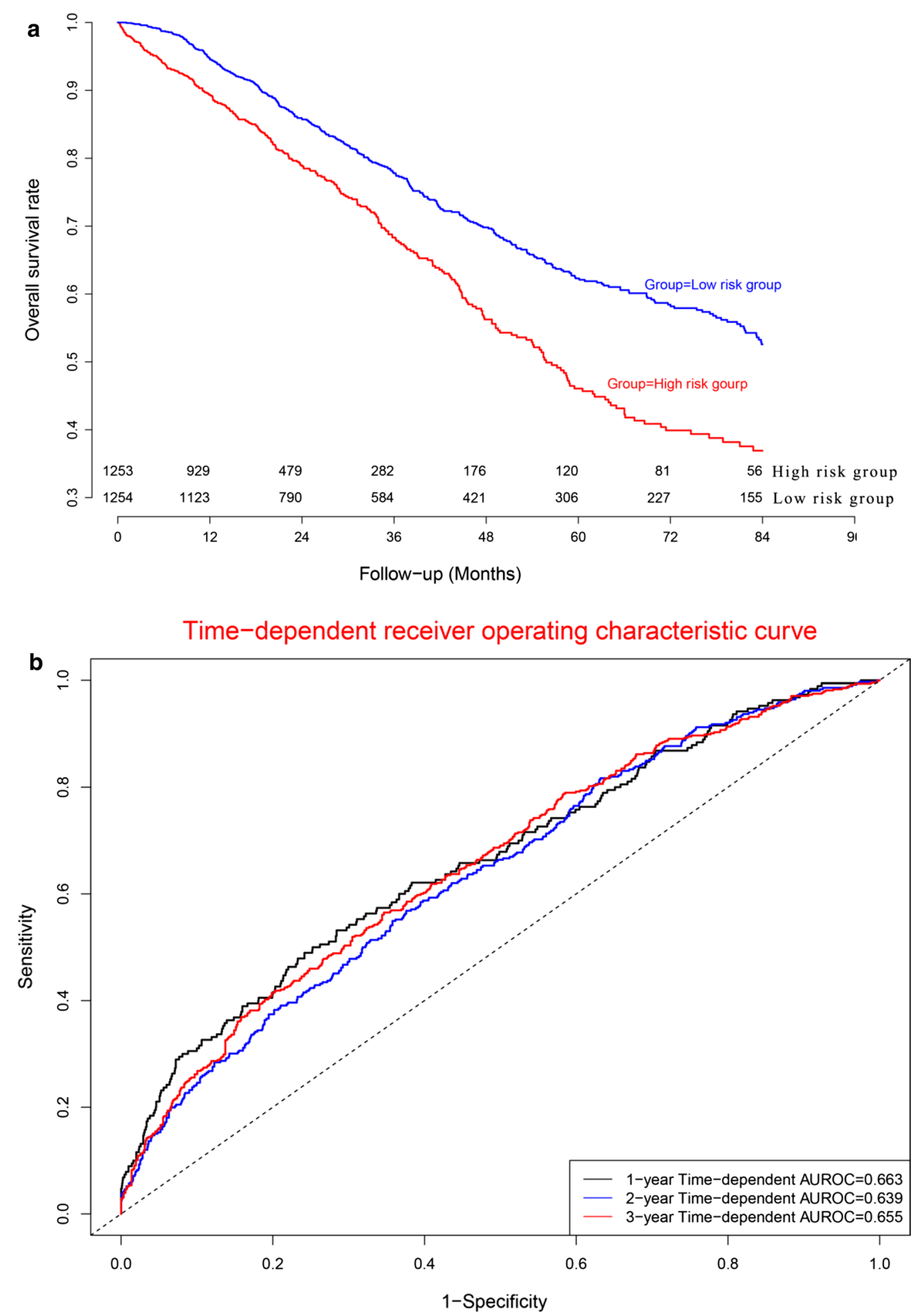

Fig. 12 Clinical performance in five malignant solid tumor datasets: a survival curves for high risk group and low risk group. b Time-dependent receiver operating characteristic curves 

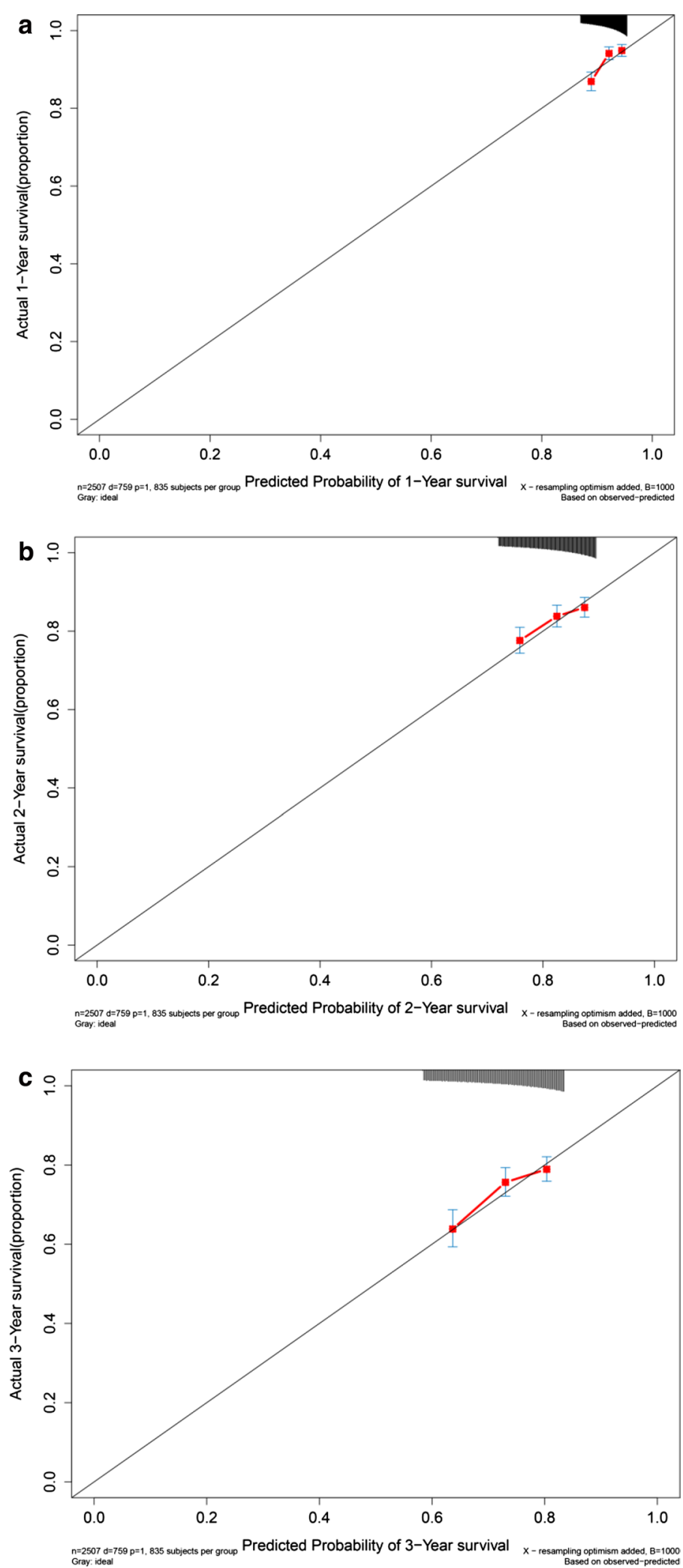

Fig. 13 Calibration curves in five malignant solid tumor datasets: a calibration curve for 1-year survival; $\mathbf{b}$ calibration curve for 2-year survival; c calibration curve for 3-year survival 
thaw until sectioned. The mirVana isolation technology uses organic extraction and then fixes RNA on glass fiber filter to purify total RNA, so it can prepare high-purity and high-quality small RNA molecules within $2 \mathrm{~h}$. RNA analytes should not undergo multiple freeze-thaw cycles to maintain biological activity. RNA is easily degraded by ribonuclease, so special methods should be taken to prevent RNA degradation. All reagents must be made of ribonuclease free materials and all products and disposable materials used must be free of ribonuclease. In order to prevent contamination by ribonuclease carried by the skin, gloves must be worn before handling biological samples. RNA analytes should be placed on wet ice and RNA quantification should be performed before freezing.

Advantages of the current study: Firstly, our study team developed a precision medicine tool named Smart Cancer Survival Predictive System, providing full-time individual mortality risk prediction with visual illustration and numerical presentation. Secondly, a novel precision medicine tool named Gene Survival Analysis Screen System was developed to explore the associations between prognostic genes (including clinical parameters) and overall survival. Users are free to choose the appropriate subgroup, gender, and stage. Meanwhile, users can upload their own dataset to explore and validate the research result.

Shortcomings of the current research: first, detection platforms were different between model dataset and validation dataset. The influence of different detection platforms on gene expression read counts should be taken into account while evaluating clinical application of the current prognostic model. Second, several important prognostic factors were not included in the current study, including surgical, radiotherapy and chemotherapy regimens. Third, the prognostic model was developed and validated by using datasets downloaded from public databases without research team's study cohort. External applicability of prognostic model needs to be validated by study cohorts from different population.

\section{Conclusions}

In conclusion, the current study provided deeper understanding of prognosis-related ceRNA regulatory network for CRC. Two precision medicine predictive tools named Smart Cancer Survival Predictive System and Gene Survival Analysis Screen System were constructed for CRC. These two precision medicine predictive tools can provide individual prognostic information before surgery and improve the individualized treatment decision-making.

\section{Supplementary information}

Supplementary information accompanies this paper at https://doi. org/10.1186/s12967-019-02151-8.

Additional file 1: Figure S1. Volcano plot: (A) Volcano plot of IncRNAs; (B) Volcano plot of miRNAs; (C) Volcano plot of mRNAs. Figure S2. Survival curves of prognostic genes. Figure S3. Decision curves and clinical impact curves.

\section{Abbreviations}

CRC: colorectal cancer; TCGA: The Cancer Genome Atlas; GEO: The Gene Expression Omnibus; ROC: receiver operating characteristic; OS: overall survival; IncRNA: long non-coding RNA; miRNA: microRNAs; HR: hazard ratio; Cl: confidence interval; AJCC: The American Joint Committee on Cancer; SD: standard deviation; DCA: decision curve analysis; ceRNA: competitive endogenous RNA.

\section{Acknowledgements}

We would like to express sincere thanks to Dr. Gary S. Collins (University of Oxford), Dr. Manali Rupji (Emory University), Mrs. Qingmei Liu for inspirations, suggestions and assistances in development of our precision medicine tools.

\section{Authors' contributions}

Conceptualization, methodology and resources: ZZ, PW, JD, LJ, LH, TH, YO, and $\mathrm{YH}$; Investigation, data curation, formal analysis, validation, software, project administration, and supervision: $Z Z, P W, J D, L J, L H, T H, Y O$, and $Y H ;$ Writing and visualization: ZZ, PW, and JD; Funding acquisition: ZZ. All authors read and approved the final manuscript.

\section{Funding}

The present study was funded by Medical Science and Technology Foundation of Guangdong Province (A2016450 and B2018237).

\section{Availability of data and materials}

The study data is available at: https://zhangzhiqiao5.shinyapps.io/Gene_Survi val_Subgroup_Analysis_B1003/.

Ethics approval and consent to participate Not applicable.

\section{Consent for publication}

Not applicable.

\section{Competing interests}

The authors declare that they have no competing interests.

Received: 21 August 2019 Accepted: 19 November 2019

Published online: 03 December 2019

\section{References}

1. Bray F, Ferlay J, Soerjomataram I, Siegel RL, Torre LA, Jemal A. Global cancer statistics 2018: GLOBOCAN estimates of incidence and mortality worldwide for 36 cancers in 185 countries. CA Cancer J Clin. 2018;68(6):394-424.

2. Kalyan A, Kircher S, Shah H, Mulcahy M, Benson A. Updates on immunotherapy for colorectal cancer. J Gastrointest Oncol. 2018;9(1):160-9.

3. Qi P, Xu MD, Ni SJ, Shen XH, Wei P, Huang D, Tan C, Sheng WQ, Zhou XY, Du X. Down-regulation of ncRAN, a long non-coding RNA, contributes to colorectal cancer cell migration and invasion and predicts poor overall survival for colorectal cancer patients. Mol Carcinog. 2015;54(9):742-50.

4. Wang X, Zhou J, Xu M, Yan Y, Huang L, Kuang Y, Liu Y, Li P, Zheng W, Liu H, et al. A 15-IncRNA signature predicts survival and functions as a ceRNA in patients with colorectal cancer. Cancer Manag Res. 2018;10:5799-806.

5. Zhang H, Wang Z, Wu J, Ma R, Feng J. Long noncoding RNAs predict the survival of patients with colorectal cancer as revealed by constructing an 
endogenous RNA network using bioinformation analysis. Cancer Med. 2019;8(3):863-73.

6. Cheng C, Wang Q, Zhu M, Liu K, Zhang Z. Integrated analysis reveals potential long non-coding RNA biomarkers and their potential biological functions for disease free survival in gastric cancer patients. Cancer Cell Int. 2019;19:123.

7. Zhang Z, Li J, He T, Ouyang Y, Huang Y, Liu Q, Wang P, Ding J. The ceRNA regulatory network reveals potential prognostic biomarkers for overall survival in hepatocellular carcinoma. Cancer Sci. 2019;110:2905-23.

8. Zeng J, Cai X, Hao X, Huang F, He Z, Sun H, Lu Y, Lei J, Zeng W, Liu Y, et al. LncRNA FUNDC2P4 down-regulation promotes epithelial-mesenchymal transition by reducing $\mathrm{E}$-cadherin expression in residual hepatocellular carcinoma after insufficient radiofrequency ablation. Int J Hyperthermia. 2018;34(6):802-11.

9. Zhong X, Long Z, Wu S, Xiao M, Hu W. LncRNA-SNHG7 regulates proliferation, apoptosis and invasion of bladder cancer cells assurance guidelines. J BUON. 2018;23(3):776-81.

10. Shi X, Zhao Y, He R, Zhou M, Pan S, Yu S, Xie Y, Li X, Wang M, Guo X, et al. Three-IncRNA signature is a potential prognostic biomarker for pancreatic adenocarcinoma. Oncotarget. 2018;9(36):24248-59.

11. Huang Y, Xiang B, Liu Y, Wang Y, Kan H. LncRNA CDKN2B-AS1 promotes tumor growth and metastasis of human hepatocellular carcinoma by targeting let-7c-5p/NAP1L1 axis. Cancer Lett. 2018;437:56-66.

12. Salmena L, Poliseno L, Tay Y, Kats L, Pandolf PP. A ceRNA hypothesis: the Rosetta Stone of a hidden RNA language? Cell. 2011;146(3):353-8.

13. Thomson DW, Dinger ME. Endogenous microRNA sponges: evidence and controversy. Nat Rev Genet. 2016;17(5):272-83.

14. Yuan W, Li X, Liu L, Wei C, Sun D, Peng S, Jiang L. Comprehensive analysis of IncRNA-associated ceRNA network in colorectal cancer. Biochem Biophys Res Commun. 2019;508(2):374-9.

15. Liang Y, Zhang C, Ma MH, Dai DQ. Identification and prediction of novel non-coding and coding RNA-associated competing endogenous RNA networks in colorectal cancer. World J Gastroenterol. 2018;24(46):5259-70.

16. Pan H, Pan J, Song S, Ji L, Lv H, Yang Z. Identification and development of long non-coding RNA-associated regulatory network in colorectal cancer. J Cell Mol Med. 2019;23:5200-10.

17. Robinson MD, McCarthy DJ, Smyth GK. edgeR: a bioconductor package for differential expression analysis of digital gene expression data. Bioinformatics. 2010;26(1):139-40.

18. Ritchie ME, Phipson B, Wu D, Hu Y, Law CW, Shi W, Smyth GK. Limma powers differential expression analyses for RNA-sequencing and microarray studies. Nucleic Acids Res. 2015;43(7):e47.

19. Jeggari A, Marks DS, Larsson E. miRcode: a map of putative microRNA target sites in the long non-coding transcriptome. Bioinformatics. 2012;28(15):2062-3.

20. Chou CH, Shrestha S, Yang CD, Chang NW, Lin YL, Liao KW, Huang WC, Sun TH, Tu SJ, Lee WH, et al. miRTarBase update 2018: a resource for experimentally validated microRNA-target interactions. Nucleic Acids Res. 2018;46(D1):D296-302.

21. Wong N, Wang X. miRDB: an online resource for microRNA target prediction and functional annotations. Nucleic Acids Res. 2015;43(Database issue):D146-52.

22. Agarwal V, Bell GW, Nam JW, Bartel DP. Predicting effective microRNA target sites in mammalian mRNAs. Elife. 2015. https://doi.org/10.7554/ eLife.05005.

23. Shannon P, Markiel A, Ozier O, Baliga NS, Wang JT, Ramage D, Amin N, Schwikowski B, Ideker T. Cytoscape: a software environment for integrated models of biomolecular interaction networks. Genome Res. 2003;13(11):2498-504.
24. Fisher LD, Lin DY. Time-dependent covariates in the Cox proportional-hazards regression model. Ann Rev Public Health. 1999;20(undefined):145-57.

25. Katzman JL, Shaham U, Cloninger A, Bates J, Jiang T, Kluger Y. DeepSurv: personalized treatment recommender system using a Cox proportional hazards deep neural network. BMC Med Res Methodol. 2018;18(1):24.

26. Xu H, Gu X, Tadesse MG, Balasubramanian R. A modified random survival forests algorithm for high dimensional predictors and self-reported outcomes. J Comput Graph Stat. 2018;27(4):763-72.

27. Nasejje JB, Mwambi H. Application of random survival forests in understanding the determinants of under-five child mortality in Uganda in the presence of covariates that satisfy the proportional and non-proportional hazards assumption. BMC Res Notes. 2017;10(1):459.

28. Alaeddini A, Hong SH. A multi-way multi-task learning approach for multinomial logistic regression. An application in joint prediction of appointment miss-opportunities across multiple clinics. Methods Inf Med. 2017;56(4):294-307.

29. Bisaso KR, Karungi SA, Kiragga A, Mukonzo JK, Castelnuovo B. A comparative study of logistic regression based machine learning techniques for prediction of early virological suppression in antiretroviral initiating HIV patients. BMC Med Inform Decis Mak. 2018;18(1):77.

30. Fang $C L$, Sun DP, Chen HK, Lin CC, Hung ST, Uen YH, Lin KY. Overexpression of mitochondrial GTPase MFN2 represents a negative prognostic marker in human gastric cancer and its inhibition exerts anti-cancer effects. J Cancer. 2017:8(7):1153-61.

31. Lin ZY, Chen G, Zhang YQ, He HC, Liang YX, Ye JH, Liang YK, Mo RJ, Lu JM, Zhuo YJ, et al. MicroRNA-30d promotes angiogenesis and tumor growth via MYPT1/C-JUN/NEGFA pathway and predicts aggres sive outcome in prostate cancer. Mol Cancer. 2017;16(1):48.

32. Liu JG, Wang HB, Wan G, Yang MZ, Jiang XJ, Yang JY. Long noncoding RNA ZFPM2-AS1 promotes the tumorigenesis of renal cell cancer via targeting miR-137. Eur Rev Med Pharmacol Sci. 2019;23(13):5675-81.

33. Li PD, Chen P, Peng X, Ma C, Zhang WJ, Dai XF. HOXC6 predicts invasion and poor survival in hepatocellular carcinoma by driving epithelial-mesenchymal transition. Aging. 2018;10(1):115-30.

34. Petraki C, Youssef YM, Dubinski W, Lichner Z, Scorilas A, Pasic MD, Komborozos V, Khalii B, Streutker C, Diamandis EP, et al. Evaluation and prognostic significance of human tissue kallikrein-related peptidase 10 (KLK10) in col orectal cancer. Tumour Biol. 2012;33(4):1209-14.

35. Xu L, Duan Y, Wang P, Yin HQ. MiR-199b-5p promotes tumor growth and metastasis in cervical cancer by down-regulating KLK10. Biochem Biophys Res Commun. 2018;503(2):556-63.

36. Liu K, Kang M, Li J, Qin W, Wang R. Prognostic value of the mRNA expression of members of the HSP90 family in non-small cell lung cancer. Exp Ther Med. 2019;17(4):2657-65.

37. Cawthorn TR, Moreno JC, Dharsee M, Tran-Thanh D, Ackloo S, Zhu PH, Sardana G, Chen J, Kupchak P, Jacks LM, et al. Proteomic analyses reveal high expression of decorin and endoplasmin (HSP90B1) are associated with br east cancer metastasis and decreased survival. PLoS ONE. 2012;7(2):e30992.

38. Kessler SM, Hosseini K, Hussein UK, Kim KM, List M, Schulthei CS, Schulz MH, Laggai S, Jang KY, Kiemer AK. Hepatocellular carcinoma and nuclear paraspeckles: induction in chemoresistance and prediction for poor survival. Cell Physiol Biochem. 2019;52(4):787-801.

\section{Publisher's Note}

Springer Nature remains neutral with regard to jurisdictional claims in published maps and institutional affiliations. 